\title{
EL ACERCAMIENTO AL TRATAMIENTO PEDAGÓGICO DE LO CORPORAL A TRAVÉS DE LA PARTICIPACIÓN EN UNA COMUNIDAD DE PRÁCTICA VIRTUAL
}

\author{
Recepción: 08/01/2019 | Revisión: 20/02/2019 | Aceptación: 25/03/2019
}

\author{
Daniel BORES GARCÍA \\ Universidad Rey Juan Carlos \\ daniel.bores@urjc.es
}

\author{
Gustavo GONZÁLEZ CALVO \\ Universidad de Valladolid \\ gustavogonzalezcalvo@gmail.com
}

\author{
Alfonso GARCÍA MONGE \\ Universidad de Valladolid \\ agmonge@gmail.com
}

Resumen: En el presente artículo de reflexión se traza el camino recorrido por un docente hasta su participación en (Re) Produce, una comunidad de práctica virtual para profesionales de Educación Física en la que, durante tres cursos escolares, intercambia experiencias, opiniones y conocimientos con varias decenas de profesores de las diferentes etapas educativas y con docentes en formación inicial. Esta vivencia permite al investigador conocer una nueva manera de entender la Educación Física, desconocida para él hasta el momento. A través de fragmentos del diario personal y de los textos de los hilos de discusión de la comunidad de práctica virtual, de donde se obtendrán los datos mediante un análisis de contenido, se recorrerá el camino del docente hasta el acercamiento al Tratamiento Pedagógico de lo Corporal, visión educativa que deja una huella indeleble en su identidad profesional. El proceso de familiarización con dicha visión será progresivo e informal, y permitirá al docente introducir en su práctica diaria aspectos como la visión global del cuerpo, la reflexión sobre la práctica, la consciencia sobre el aprendizaje y el diseño curricular abierto y cambiante.

Palabras clave: Educación Física; comunidad de práctica virtual; Tratamiento Pedagógico de lo Corporal.

THE APPROACHTOTHE PEDAGOGICAL TREATMENT OFTHE BODYTHROUGH PARTICIPATION IN A VIRTUAL COMMUNITY OF PRACTICE

\begin{abstract}
This paper traces the path traveled by a teacher to his participation in (Re)Produce, a virtual community of practice for Physical Education professionals in which, during three school years, he exchanges experiences, opinions and knowledge with several dozen teachers of the different educational stages and with teachers in initial training. This experience allows the researcher to get to know a new way of understanding Physical Education, unknown to him until that moment. Through fragments of his personal diary and the texts of the discussion threads of the virtual community of practice, where the data will be obtained through content analysis, the path of the teacher will be covered until reaching the approach to the Pedagogic Treatment of the Body, an educative view that leaves an indelible mark on his professional identity. The process of familiarization with this vision will be progressive and informal and will allow the teacher to introduce into his daily practice aspects such as the global vision of the body, reflection on practice, awareness of learning and open and changing curricular design.
\end{abstract}

Keywords: Physical Education, virtual community of practice, Pedagogic Treatment of the Body.

\section{L'APROPAMENT AL TRACTAMENT PEDAGÒGIC DEL COS ATRAVÉS DE LA PARTICIPACIÓ EN UNA COMUNITAT DE PRÀCTICA VIRTUAL}

Resum: En el present article de reflexió es traça el camí recorregut per un docent fins a la seva participació a (Re)Produce, una comunitat de pràctica virtual per a professionals d'Educació Física en la que, durant tres cursos escolars, intercanvia experiències, opinions $i$ coneixements amb diverses desenes de professors de les diferents etapes educatives i amb docents en formació inicial. Aquesta vivència permet a l'investigador conèixer una nova manera dentendre l'Educació Física, desconeguda per a ell fins llavors. A través de fragments del diari personal i dels textos dels fils de discussió de la comunitat de pràctica virtual, don sobtindran les dades mitjançant una anàlisi de contingut, es recorrerà el camí del docent fins a lapropament al Tractament Pedagògic del Cos, visió educativa que deixa una marca indeleble en la seva identitat professional. El procés de familiarització amb aquesta visió serà progressiu i informal, i permetrà al docent introduir en la seva pràctica diària aspectes com la visió global del cos, la reflexió sobre la pràctica, la consciència sobre l'aprenentatge $i$ el disseny curricular obert $i$ canviant.

Paraules clau: Educació Física; comunitat de pràctica virtual; Tractament Pedagògic del Cos. 


\section{Introducción}

En la búsqueda de nuevas ideas pedagógicas y prácticas educativas con potencial para mejorar la práctica docente (Armour y Yelling, 2004), durante tres cursos escolares varios profesionales de la Educación Física provenientes de diferentes contextos laborales (educación primaria, educación secundaria y educación universitaria) y un buen número de docentes en formación inicial participaron en (Re)Produce, una comunidad de práctica virtual (Lave y Wenger, 1991; Wing Lai et al., 2006) alojada en la plataforma MultiScopic (González Calvo y Bores García, 2014; Bores García, González Calvo y García Monge, 2018; García Monge, González Calvo y Bores García, 2018) analizando prácticas compartidas en el contexto escolar. Esta comunidad de práctica, con un total de 63 participantes, permitió que se generaran multitud de diálogos en formato de foro en los que se compartió conocimiento sobre la Educación Física escolar a partir de experiencias de unidades didácticas relacionadas con la estrategia en el juego, la carrera, el parkour, el acrosport o la danza de contacto, entre otros contenidos.

El germen de la plataforma MultiScopic se encuentra en la Escuela Universitaria de Educación (EUE) de Palencia en la que en 1989 se pone en marcha un seminario de formación permanente denominado Tratamiento Pedagógico de lo Corporal (Vaca Escribano, 2007, 2010) en el que, siguiendo procesos de investigación-acción sobre la práctica educativa, profesorado de diferentes niveles educativos intentaba desarrollar una idea en torno al valor del cuerpo y la motricidad en la enseñanza obligatoria. Un año más tarde en ese centro universitario se comenzaría a formar a la primera promoción de maestros especialistas en Educación Física en España. Desde entonces, muchos han sido los maestros participantes en el Seminario Tratamiento Pedagógico de lo Corporal (TPC) y los estudiantes universitarios formados en la misma forma de entender la Educación Física Escolar (Bores Calle, 2005; Martínez Álvarez et al., 2009).

Para poder compartir una idea, primero hay que tenerla clara. Para que el TPC pudiera abrirse a un espacio on-line en el que los matices son difíciles de explicar y el contacto puede ser fugaz, el grupo debía definir claramente sus ideas sobre la persona, la educación o la Educación Física. Rastreando sobre el proceso de creación de MultiScopic encontramos que los componentes del grupo InCorpora dedicaron un año de debates semanales a la puesta en común de sus "señas de identidad" (curso 2009-2010). En 24 años de trabajo, son múltiples las ideas que un grupo va compartiendo y el conocimiento que va construyendo, pero no siempre éste conforma un todo homogéneo, coherente y explícito; algo que nos recuerda a la idea que Bruner (1996) toma de Wittgenstein sobre los conceptos como construcciones de múltiples fibras. Al revisar las actas de dichas reuniones constatamos:

- La diversidad de temas y la ramificación de los mismos. El grupo comienza intentando responder a las preguntas básicas sobre cómo entiende a la persona, la sociedad, el aprendizaje, la educación, la escuela y la Educación Física. Los componentes del grupo InCorpora han bebido de fuentes pedagógicas, psicológicas, antropológicas, sociológicas y filosóficas diversas y no siempre es posible casar posturas alejadas o antagónicas. Apreciamos un conocimiento construido a retazos en el que hay unas ideas genéricas básicas comunes, pero con múltiples 
incoherencias fruto de un bagaje que ha ido evolucionando e incorporando nuevas perspectivas y preocupaciones profesionales.

- Los matices y sensibilidades particulares entre los miembros de InCorpora en un conocimiento que parecía homogéneo y compartido. El análisis de las actas de estas reuniones nos ilustra también sobre las divergencias en las construcciones personales del conocimiento a lo largo de 24 años de formación compartida. Se aprecia cómo el conocimiento compartido se construye individualmente con matices y énfasis personales.

- La falta de respuesta o incoherencia ante preguntas básicas sobre persona, educación, educación física, etc. El conocimiento del grupo se ha ido construyendo a través de unos supuestos teóricos genéricos, lejanos; y a partir de múltiples experiencias personales ligadas a la práctica educativa y su posterior análisis. Ello hace que se genere una amplio y detallado corpus teórico ligado a la planificación-desarrollo y evaluación de situaciones educativas, pero quedan muchos resquicios en el gran mosaico del conocimiento. Se han generado teorías abarcadoras (Schön, 1998), conceptos sensitivos (Blumer, 1982), hipótesis de trabajo y teorías de alcance intermedio (Merton, 1980) que ayudan al grupo a orientarse en las propuestas prácticas, pero que no siempre les permiten dar respuesta a múltiples cuestiones sobre la educación ni generar un discurso pedagógico más global.

A partir de estos debates, los miembros del grupo InCorpora llegaron a unos acuerdos básicos sobre sus señas de identidad y los fundamentos de su propuesta educativa, de manera que se podría decir que el diseño de un proyecto de este tipo brinda una oportunidad para reforzar una identidad grupal y explicitar el conocimiento tácito, aunque puede generar tensiones que fragmenten al grupo.

Tras la explicación de la metodología utilizada, en el epígrafe 2 trataré de describir la forma en la que tomé contacto con una manera de ver la enseñanza de la Educación Física desconocida para mí a través de la experiencia en la comunidad de práctica (Re)Produce, que sirvió como campo de trabajo para la redacción de mi tesis doctoral, defendida en diciembre de 2016. Pero para entender este proceso creo preciso comenzar con una breve historia de mi trayectoria académica y profesional, acabando con los elementos que del TPC pude al final incorporar a mi práctica docente hasta hoy. Para ello me serviré de extractos de un diario personal que escribí durante los tres cursos de la experiencia y de algunos fragmentos de los hilos de discusión del grupo (Re)Produce. Para asegurar el anonimato de los participantes en la comunidad de práctica, se ha asignado un número a cada uno de ellos. Tampoco aparecen los nombres reales durante el texto, sino solamente la inicial.

\section{Metodología}

Se han utilizado dos instrumentos para la recogida de datos. Por un lado, el docente que protagoniza la experiencia elabora durante la misma un diario personal (Zabalza, 2008) en el que recoge sus sensaciones y reflexiones más destacadas, mediante una autoetnografía que permite la explo- 
ración y profundización en los procesos reflexivos a través de su evolución personal y profesional (Segelström y Holmlid, 2015). Por otro lado, se lleva a cabo un análisis de contenido de los distintos hilos de debate producidos en el grupo (Re)Produce, alojado en la plataforma MultiScopic, entre los años 2013 y 2015, que complementan la información obtenida del análisis del diario del docente. Además, para contextualizar la situación del docente con respecto a la visión pedagógica a la que se hace referencia en el presente artículo, se esboza una breve historia de vida del docente.

\section{Mi acercamiento al TPC}

Puesto que la enseñanza es algo personal, para conocer cómo es la enseñanza es necesario conocer cómo es el docente (González-Calvo y Arias-Carballal, 2017; González-Calvo y FernándezBalboa, 2016; González Calvo, 2013; Goodson, 2004). Devís y Sparkes (2004, p.87) señalan que esta identidad profesional "es algo social, múltiple, fragmentado, cambiante, emergente, ambiguo, contradictorio, contextual, relacional y distribuido a lo largo del tiempo y del espacio". Son muchos los factores que conforman la personalidad de un docente y su forma de comportarse en el aula y entender su profesión, y muchos de ellos están ligados a aspectos sociales y personales (GonzálezCalvo y Arias-Carballal, 2017; González-Calvo y Fernández-Balboa, 2016; González Calvo, 2013; Nias, 1989). Mi comportamiento en mi profesión y en Multiscopic vendrá, pues, determinado en gran medida por mi identidad profesional y ella, en parte, por mis experiencias formativas y profesionales. Con la convicción de que una mejor comprensión de mis antecedentes formativos hará más claro el entendimiento de mi participación en Multiscopic, a continuación haré un breve recorrido por los principales hitos en mi formación, poniendo especial énfasis en los elementos más notables del Tratamiento Pedagógico de lo Corporal, una forma de entender la Educación Física que se haya presente en todos los comentarios de (Re)Produce y que conforma la base conceptual de los diseños de las Unidades Didácticas que se han replicado en el grupo virtual. Uno de los objetivos más importantes de esta pequeña historia de mi vida académica será explicar en qué medida han influido en mi identidad profesional los aprendizajes adquiridos en los diferentes estudios realizados y de qué manera pude comenzar a integrar a la misma un modelo pedagógico novedoso para mí como es el TPC.

\subsection{La Facultad de Ciencias de la Actividad Física y del Deporte}

Mi formación como docente se inicia en el año 2005 en la Facultad de Ciencias de la Actividad Física y del Deporte (INEF, Universidad Politécnica de Madrid). Allí termino licenciándome en julio del año 2010. Durante esta etapa se hace fuerte la opción de dedicarme a la docencia de la Educación Física, idea que ya viene desde la edad de catorce años. En lugar de matricularme en alguna de las maestrías deportivas en los dos últimos cursos, opto por cursar asignaturas relacionadas con la educación. En el último año de la carrera hago las prácticas educativas en el Instituto Alameda 
de Osuna de Madrid, experiencia totalmente positiva y enriquecedora para mí gracias a la figura de mi profesor de prácticas. En tan solo unos meses descubro una forma de hacer Educación Física que me impacta, fundamentalmente aquellos contenidos propios del bloque de Expresión Corporal. En aquel instituto, particularmente en $1^{\circ}$ de Bachillerato, la danza era un contenido que inevitablemente todo alumno debía experimentar de una manera radicalmente distinta a lo que yo estaba acostumbrado a ver: clases en las que el propio profesor, que superaba la cincuentena, enseñaba a los alumnos a bailar rock, salsa, merengue, bachata, etc. Al final de la Unidad Didáctica, por grupos, los alumnos hacían una representación bailada utilizando todos los elementos que habían aprendido. Chicos y chicas de dieciséis años, fuera cual fuese su contexto y su actitud frente al instituto, se implicaban al cien por cien en esta Unidad Didáctica. Aquello se me quedó grabado y aprendí que cualquier contenido de Educación Física, si se le da una orientación correcta, puede resultar relevante y estimulante para el alumnado. Asignaturas como Metodología I, Metodología II, Pedagogía, Aprendizaje Motor, Educación Física de Base, etc. dejaron poca huella, al menos consciente, en mi formación. Años después, mientras escribo estas líneas, pienso, no sin cierta tristeza, que son pocas cosas las que recuerdo de aquellas clases. No puedo identificar una forma de entender la Educación Física o una línea de actuación docente común a todo el profesorado de la Facultad. Ni siquiera recuerdo que me resultara novedoso lo que escuchaba en aquellas clases o lo que observaba en las lecturas recomendadas. Más bien tengo la sensación de haber estudiado a Piaget cinco veces por cada vez que estudiaba alguna pedagogía innovadora.

\subsection{El Máster en Formación del Profesorado de Enseñanza Secundaria y Bachillerato}

Durante mi último curso en el INEF comenzamos a escuchar voces que decían que ya no habría Curso de Adaptación Pedagógica (CAP) al año siguiente. Esto quería decir que los que habíamos tomado la orientación hacia la educación teníamos un serio problema. Se oía que, en lugar del CAP, habían creado un Máster Universitario Oficial de 60 créditos cuya obtención acreditaba lo mismo que el antiguo CAP. Y efectivamente, mi promoción fue la primera que estuvo obligada a cursar el Máster en Formación del Profesorado de Enseñanza Secundaria y Bachillerato, al menos quien quisiera presentarse a oposiciones o solicitar un puesto de trabajo en cualquier colegio Privado o Concertado. Después de examinar las diferentes opciones, me matriculé del Máster en la Universidad Autónoma de Madrid. Allí, dejando a un lado las mil y una redundancias de las mil y una asignaturas redundantes que tuvimos que estudiar, me dejó marcado un profesor: Juan Luis Hernández Álvarez. Sus clases de Didáctica supusieron para mí el primer acercamiento a la legislación sobre educación en toda mi formación. Por vez primera pude enfrentarme a la tarea de programar partiendo de la referencia de la ley (la LOE en aquel momento). En clase examinábamos cada uno de los objetivos generales, de los que sacábamos los objetivos específicos que más tarde debíamos usar como justificación para cada una de las tareas propuestas en las sesiones de las Unidades Didácticas. Aquel profesor me hizo ser consciente de la importancia de la legislación como elemento dentro del cual se enmarca nuestra profesión. Con todo, salvo una clase en la que 
recuerdo hablar de cómo abordar en una Unidad Didáctica un contenido de desarrollo de las capacidades físicas básicas, los aprendizajes que extraje de aquel Máster se quedaron en un terreno demasiado genérico, para mi gusto. Fueron pocas las ocasiones que tuvimos de ponernos a pensar en el día a día del aula, en las programaciones, en cómo llevar a cabo una Unidad Didáctica, en cómo establecer el control en una clase, cómo gestionar los grupos, cómo abordar el trabajo con el cuerpo. Las prácticas educativas, que eran una asignatura obligatoria del Máster, las hice en un centro Concertado con un profesor con una forma de trabajar muy orientada al deporte reglado (fundamentalmente fútbol y baloncesto), por lo que no tuve ocasión de observar sesiones de Expresión Corporal, Habilidades Motrices, actividades de reflexión sobre la táctica o la estrategia, contenidos relacionados con el diseño de programas de salud, etc.

Hay ciertas carreras universitarias en las que el lugar en el que se estudian resulta determinante para el tipo de formación que se tendrá al terminar. Hay facultades con un marcado estilo o forma de entender una rama del saber en concreto. Pero, por las razones que fueran, si esto puede ocurrir con los estudios de Educación Física, yo no tuve la ocasión de estudiar en ninguna de ellas.

\subsection{El Magisterio con la especialidad en Educación Física}

En la misma época en la que se oía hablar de la inminente retirada del CAP y la más que segura necesidad de cursar el Máster en Formación del Profesorado, también se comentaba que en ciertas universidades privadas existía la opción de cursar Magisterio con la especialidad de Educación Física asistiendo a clase durante un solo año, durante los fines de semana. Esto era debido a la gran cantidad de asignaturas que, por ser Licenciado en Ciencias de la Actividad Física y del Deporte, podían ser convalidadas. Así pues, en el curso 2010/2011 me matriculé simultáneamente del Máster y de Magisterio. Este último lo cursé en la Universidad Camilo José Cela. La forma en la que se estructuraban los estudios para aquellos alumnos licenciados era en un formato semipresencial. Los sábados había seis horas de clase en las que estudiábamos aquellas materias más propias del Magisterio en general, tales como Matemáticas, Conocimiento del Medio, Lengua, Psicología, Didáctica General, Historia de la Educación, Organización del Centro Escolar, etc. De esta forma, si bien algunas asignaturas que cursé fueron muy valiosas para mi formación general, la específica no se enriqueció ya que todas las asignaturas referidas a la Educación Física en particular estaban convalidadas con mi Licenciatura. No sé si aquella Universidad tenía una concepción particular de la enseñanza de la Educación Física, pero yo no tuve siquiera la oportunidad de comprobarlo.

\subsection{Los inicios en el terreno profesional}

En julio del año 2011, el mismo mes en el que terminé mis estudios de Magisterio y del Máster, hice una entrevista en un colegio Concertado de Madrid y me contrataron como profesor de Música a media jornada en la etapa de Primaria. Mi Título Profesional de Música, junto con la Diplo- 
matura en Magisterio, me habilitaba para ello y, ya que en los últimos años no había recibido una formación específica en relación a la didáctica de la Música, tuve que comenzar a investigar por mi cuenta de qué forma impartir la asignatura. Fue un año muy positivo porque pude conocer cómo se trabaja en un colegio, asistí a mis primeras sesiones de evaluación con todo el claustro, experimenté lo que es trabajar dentro de un equipo de personas muy diversas, etc. El curso siguiente me concedieron por fin la jornada completa y, por primera vez, fui profesor de Educación Física. Me asignaron un grupo de $1^{\circ}$ de Primaria, otro de $4^{\circ}$ y uno de $6^{\circ}$. En aquel colegio en ese momento éramos siete profesores de Educación Física; por lo tanto, nos repartimos el trabajo para diseñar entre todos las Unidades Didácticas de los distintos cursos. Desde el primer momento detecté una orientación demasiado deportivista para mi forma de entender la Educación Física. Yo venía de haber estado estudiando la legislación en el Máster y de ver cómo los juegos y deportes eran tan solo una cuarta o quinta parte de los contenidos de la materia. Tuve que luchar por conseguir que se le asignara un hueco en la programación a contenidos de Expresión Corporal, por ejemplo. Y, desde el primer día, también me encontré en un continuo intento por contrarrestar las experiencias previas de los alumnos, acostumbrados a jugar al baloncesto y al fútbol en clase de Educación Física. El año siguiente también tuve algún grupo al que daba Educación Física, y mi marco de trabajo fue mi propia labor del curso anterior y el tratar de solventar los errores que yo mismo había cometido el año anterior o que había visto cometer al resto de profesores. No encontré a ningún profesor que pudiera servirme como referencia o modelo a seguir. Lo que sí encontré fueron modelos de lo que estaba seguro de no querer reproducir como profesional.

\subsection{La irrupción delTPC en mi camino}

Durante mi segundo año en el colegio en el que trabajaba, la idea de hacer la Tesis Doctoral ya rondaba mi cabeza y había tenido los primeros contactos con algunos profesores universitarios en Valladolid con los que hice un pequeño sondeo de un buen tema para la posible investigación. Uno de estos profesores me habló de un colega de la universidad que había empezado una plataforma online, a modo de experiencia piloto, y que quizá podría servir como campo de investigación de diversos temas relacionados con la Educación Física. Me invitaron a una conferencia en Palencia con muchos otros profesores universitarios de Educación Física en la que pude tener mi primer contacto tanto con Multiscopic como con el TPC. El funcionamiento de Multiscopic lo entendí bastante rápido, pero no ocurrió lo mismo con el Tratamiento Pedagógico de lo Corporal. Los participantes en Multiscopic daban por hecho, en sus conversaciones, que el TPC era algo conocido por todos. Yo, al menos, no lo había oído nunca. Me di cuenta de que el nacimiento de esta corriente estaba en la Universidad de Valladolid, en la que yo nunca he realizado estudio alguno. Esto me tranquilizó un poco; también caer en la cuenta de que casi todas las personas relacionadas con el TPC habían pasado por las aulas de la Universidad de Valladolid o, al menos, habían tenido algún contacto con aquellos Seminarios que organizaban algunos profesores de la Universidad y en los que, entre otras cosas, comenzó el germen de esta forma de entender y enseñar la Educa- 
ción Física.

Mi primera lectura sobre el TPC fue en Multiscopic, en el apartado en el que se explica en qué consiste esta corriente. Aún así, no fue hasta que yo mismo comencé a participar en Multiscopic cuando de verdad pude empezar a empaparme del TPC y a conocer su lado más práctico y con más aplicabilidad en el aula. Algunas expresiones como "que aprendan siendo conscientes de su aprendizaje", "huir del dualismo cuerpo-mente", "que apunten en el cuaderno", "en la puesta en común de la práctica", "en el tiempo de reflexión” ... se me antojaban mantras que los participantes en Multiscopic repetían con total naturalidad. Y para mí algunos de ellos eran totalmente sorprendentes.

\subsection{Mi experiencia con el Tratamiento Pedagógico de lo Corporal en (Re) Produce}

Los inicios del grupo (Re)Produce hacían ya pensar que el grueso de sus miembros guardaba una fuerte relación con el TPC. Y conforme fueron creándose los hilos de debate, este hecho se confirmó. La realidad es que, de los miembros con mayor número de comentarios en el grupo, yo era el único cuyo origen estudiantil y profesional no estaban relacionados con esta forma de entender la Educación Física. A lo largo de los dos cursos en los que se han producido las interacciones vía online en la comunidad de práctica, muchos han sido los conceptos y expresiones con las que he tenido que irme familiarizando y cuyo significado he ido descodificando con la ayuda de los otros participantes, del contexto, de la lectura de los comentarios, del visionado de los vídeos y de las charlas presenciales con algunos de ellos (fundamentalmente con mis directores de Tesis).

a) La desaparición de la dualidad cuerpo-mente

Mi formación como docente de Educación Física se ha basado en un axioma, inconsciente y sumergido en el currículum oculto de muchos de mis profesores en mi etapa primaria, secundaria y también en la universidad, que separaba al individuo en cuerpo por un lado y mente por otro. El cuerpo hacía referencia a los aprendizajes de tipo motor tales como habilidades motrices básicas, capacidades físicas básicas, la práctica predeportiva y deportiva, etc. mientras que la mente tenía más que ver con los aprendizajes relacionados con lo ético, lo social, la aceptación de las limitaciones y posibilidades, la asimilación de los conceptos teóricos, la táctica en los deportes, etc. Tanto es así que al hablar de contenidos seguíamos hablando de contenidos actitudinales y conceptuales (relacionados con la mente) y contenidos procedimentales (relacionados con el cuerpo). Al iniciar mi participación en (Re)Produce, rápidamente noté que de forma transversal a todos los hilos de discusión en ningún momento se produce una separación entre lo teórico y lo práctico, tratando de que justamente las sesiones se diseñen para incidir en los aprendizajes de los alumnos desde el punto de vista holístico.

Hasta ahora, lo que más me ha llamado la atención ha sido la forma integradora que tiene de ver al sujeto. Lo mental, lo emocional, lo relacional y lo corporal son todo uno, pues el alumno es todo esto conviviendo en una misma esencia. Todavía no sé las repercusiones 
prácticas que tiene esta forma de ver al individuo, pero sí que voy viendo que la reflexión sobre la práctica es fundamental en el TPC. (Diario personal del investigador. 8 de enero de 2014).

No obstante, y pese a saber esto de forma teórica, en varias ocasiones algunos participantes en (Re)Produce han identificado en mis discursos expresiones que aludían inconscientemente a esta división cuerpo/mente.

Por hacer un simple comentario a D., cuando dices en un momento dado "aquí entra de nuevo el aspecto reflexivo, incluyéndose en el cuerpo del alumno también su mente", yo lo explicaría de otra manera. Más que nada porque, con afirmaciones de ese tipo, seguimos perpetuando esa idea dualista de persona compuesta por "diferentes partes". Idea que, además, en poco favorece a la Educación Física, desde mi punto de vista. (Comentario de participante 3 en (Re)Produce, 12 diciembre 2013)

b) La consciencia sobre el aprendizaje

Uno de los pilares del TPC con el que me topé desde un principio fue el del aprendizaje consciente. Hasta entonces, muchas de las tareas que yo había visto diseñar y poner en práctica en clases de Educación Física estaban orientadas a que el alumno aprendiera sin darse cuenta. Se trataba, pues, de concatenar una serie de ejercicios con un objetivo compartido que solo conocía el profesor y que los alumnos no tenían por qué conocer, porque lo importante era que alcanzaran el objetivo, no que lo conocieran.

Un concepto clave que yo creo que es esencial en el TPC es que el alumno SÍ debe ser consciente del proceso en el que está inmerso. La idea de que "el alumno aprenda sin darse cuenta" es desterrada en este modelo, algo que puede chocar a quien académicamente o profesionalmente ha estado imbuido (inconscientemente quizá) de esta idea. Me parece muy adecuado el axioma de que el alumno debe participar en el proceso de enseñanzaaprendizaje sabiendo el rol que ocupa, para qué sirve cada elemento del proceso y cuál es el objetivo que se quiere alcanzar. Esto fomenta el trabajo de reflexión por parte del alumnado $y$ el concepto de transferencia y aplicabilidad de los aprendizajes. (Primer comentario de participante 1 el en el hilo de "Juego Bueno". 22 de noviembre de 2013).

Aquí entra otro punto fundamental del TPC que es la idea de que el alumno sepa qué debe aprender y cómo lo está aprendiendo (el alumno como centro del aprendizaje, consciente de los procesos que le llevan a él). Esto me gusta, porque aleja la Educación Física de una idea ocultista que no hace sino restar prestigio a la disciplina. El alumno debe conocer qué va a aprender y cuáles van a ser los mecanismos que le van a conducir a tal aprendizaje. Así pues, la competencia de aprender a aprender se desarrolla de una forma mucho más explícita. (Diario personal. 8 de enero de 2014).

Estas reflexiones, muy al inicio del proceso, me preparaban ya para poner en práctica yo mismo las Unidades Didácticas propuestas por P. en el colegio de Frómista en el que trabajaba. 
Buscar que el alumno supiera en todo momento qué estaba aprendiendo y para qué era un reto importante que influiría mucho en mi manera de diseñar las sesiones. Al comienzo no fue sencillo, ya que para mí era complicado lograr aprendizajes conscientes sin detener demasiado el ritmo de la clase. Algunos de los alumnos me decían, sobre todo al principio: “YYa estamos parando otra vez!", “'Qué pesado con lo de los objetivos de la lección!”, etc.

Penso de qué forma podría haber introducido elementos de movimiento corporal en esta segunda sesión sin renunciar al proceso reflexivo y de trabajo en equipo compartiendo opiniones y llegando a acuerdos de forma grupal. (Comentario de participante 1 en (Re)Produce, 27 noviembre 2013)

Estas quejas de los alumnos, acostumbrados por otra parte a realizar automáticamente tareas para las que no conocían el objetivo, me hacían reflexionar sobre la conveniencia de incidir tanto en este aspecto. Quizá tenía miedo a que los alumnos se desengancharan del proceso por sentirse abrumados.

Estoy dándole vueltas a la forma de explicitar qué quiero que aprendan. No quiero desviarme del TPC, sino que quiero justamente hacer conscientes a los alumnos del proceso en el que están. Pero no quiero dar discursitos entre ejercicio y ejercicio. (Comentario de participante 1 en (Re)Produce, 21 febrero 2014)

Este sentimiento de incompatibilidad entre el aprendizaje consciente y la motivación del alumno fue desapareciendo a medida que me di cuenta de que la tensión entre ambos elementos podía minimizarse si se hacía un correcto tratamiento pedagógico.

Actualmente, en mis clases de Educación Física, utilizo el tiempo de movilidad articular al inicio de la lección para ir introduciendo al alumnado en los contenidos que se trabajarán durante la misma y para establecer cuál es el objetivo (o los objetivos) que ellos mismos deben tratar de alcanzar en ella. De esta manera no entran en conflicto temporal la práctica y la teoría y los alumnos comprenden desde el inicio qué se pretende con la lección. Otro ejemplo que ilustra mi cambio hacia un aprendizaje mucho más consciente está en la ficha de observación que entrego a los alumnos que, por razones de enfermedad o lesión, no pueden desarrollar las tareas programadas para la lección con el resto de la clase. Les pido que observen lo que hacen sus compañeros y que vayan anotando y dibujando cada tarea. Una de las peticiones que les hago al final de la ficha es que traten de explicar cuál ha sido el objetivo de la lección, qué aprendizajes se pueden extraer de la misma. De esta forma les "obligo" a pensar no solo en qué actividades se han hecho sino también, que es lo más importante, para qué se han hecho y qué se ha podido aprender con ellas.

c) El docente como provocador de situaciones de reflexión

En línea con el apartado anterior, el TPC da una enorme importancia a la reflexión del alumno de forma individual y colectiva acerca de las prácticas que realiza en las sesiones. El profesor, por lo tanto, ya no tiene el rol de programar y dirigir actividades con un objetivo general y dos o tres específicos, sino que tiene la responsabilidad de diseñar actividades que generen situaciones de las 
que los alumnos puedan extraer aprendizajes que vayan más allá de la actividad y sean extrapolables a situaciones diferentes.

...pienso que el aspecto de la reflexión acerca de la seguridad es algo a lo que el TPC da mucha importancia, mientras que otras corrientes incidirían mucho más en la práctica motriz. He planteado al grupo cómo podrían combinar estos dos aspectos, pues pienso que en esta UD lo que a los alumnos les gusta es "hacer", más que "pensar". Me sigue costando un poco la idea de una Educación Física con menos compromiso motor. Me gusta la idea en la teoría, pero a la hora de enfrentarme a la clase, sufro cada vez que tengo que parar la práctica, porque pienso que paro para "soltar un rollo". Seguimos adelante, no obstante. (Diario personal. 10 de febrero de 2014).

No obstante, uno de los participantes en (Re)Produce familiarizado desde hace años con el TPC, al leer este fragmento del diario personal del investigador, expone lo siguiente:

Esto no es del todo así. Intentamos que la reflexión sirva para mejorar la acción, para darle sentido. No se trata de reflexionar por que sí. Por otra parte, intentamos que haya el mayor tiempo de práctica posible. Pero el objetivo no es el tiempo de movimiento sino la calidad del aprendizaje que se produce. Lo ideal es que la reflexión esté integrada en la acción. Buscamos que las propuestas tengan sentido para los escolares por que la reflexión les ayuda en su proceso de aprendizaje. Para un niño tiene mucho sentido hacer una estrategia junto a otro, u orientar un plano chino ante un recorrido. Por ejemplo, podemos ponernos música para correr y "desconectar" de la fatiga o correr conscientemente atendiendo a las señales del esfuerzo. (Comentario de matización de participante 4 en (Re)Produce. Julio de 2016)

Aquí entran en juego las experiencias previas tanto del profesor como del alumno. Si, tanto en un rol como en otro, uno está acostumbrado a sesiones en las que el tiempo de compromiso motor (Sierra, 2000, 2003) o la participación activa (Generelo, 1995) ocupa la mayor parte de las mismas, resultará un verdadero desafío detener la acción motriz para abrir un tiempo de reflexión grupal o para que el alumno trabaje en su cuaderno, tal como sugiere Bores (2006) y que ya se ha convertido en una práctica habitual en muchos centros educativos. Reconocí desde el primer momento una resistencia por parte del alumnado a los tiempos de reflexión, ya que estaban acostumbrados a no parar de moverse desde el comienzo hasta el final de las sesiones en cursos anteriores. Mi reto fue lograr momentos de reflexión relevantes para el alumno, de tal forma que no fueran considerados como un castigo o un mal trago, sino como un momento necesario para poner en común los aprendizajes y para obtener diversos puntos de vista de otros compañeros y del profesor.

Al finalizar la sesión, el profesor solicitará a los alumnos que reflexionen acerca de las alternativas/modificaciones que han hecho, con cuál se han divertido más, cuál es la que menos les ha gustado, si se les ocurren más alternativas, etc... (Comentario de participante 1 en (Re)Produce, 27 noviembre 2013) 
En un centro educativo con siete profesores de Educación Física, seis de ellos fuertemente ligados a modelos pedagógicos más tradicionales en los que los procesos reflexivos no estaban casi presentes, quizá el principal obstáculo no era yo mismo, aunque como he explicado anteriormente tuve que convencerme a mí mismo que la reflexión sobre la práctica era esencial en la Educación Física, sino las experiencias previas de los alumnos. Acostumbrados a la concatenación sin descanso de tarea tras tarea (más bien de juego tras juego), presentaban reticencias a las paradas para reflexionar sobre lo que hacíamos. Relacionaban aula con reflexión y gimnasio con movimiento, sin entender que el gimnasio es un aula más en el que también se aprenden cosas. Hicieron falta varios meses para que los alumnos fueran asimilando mi forma de trabajar y acostumbrándose a los tiempos de reflexión.

11:00. Los alumnos están en fila en el patio (acaba de terminar el recreo) y yo les digo que subimos a clase. Esto supone un contratiempo para ellos y me lo hacen notar con frases como: “¿Por qué?”“¿No va a haber educa?” ¿No vamos a ir al poli?”“¿Pero qué hace?”... (Extracto de comentario de participante 1 en (Re)Produce, 27 noviembre 2013)

El alumno sabe que tal día a tal hora, justo después del recreo, hay Educación Física. Lo habitual es que el profesor mande a la fila directamente al gimnasio para comenzar la clase. Es una sorpresa, entonces, que ese día el profesor les diga que van a clase, como si en lugar de Educación Física tuvieran Lengua o Matemáticas. Las expresiones, mezcla entre sorpresa y contrariedad, de los alumnos reflejan perfectamente cómo llega el mensaje a los alumnos. Este hecho puntual ilustra una realidad generalizada en aquellos grupos acostumbrados a un tipo de Educación Física y que se muestran poco flexibles a cambios de cualquier tipo, máxime cuando estos cambios reducen el tiempo de esparcimiento que los alumnos esperan del período de Educación Física. Por ello señalo que la forma en la que los procesos reflexivos fueron quedando más normalizados no tuvo que ver tanto con acciones metodológicas concretas sino con el mantenimiento en el tiempo de una nueva forma metodológica que al final, como suele suceder con los actos repetidos, generó un hábito. No obstante, siendo sincero tengo que decir que cuando finalizó el curso los alumnos seguían sin implicarse del todo en los tiempos de reflexión; más bien se mostraban impacientes durante los mismos esperando a que se terminaran para poder seguir realizando los ejercicios. Posiblemente, no logré dar sentido a la reflexión y la consciencia sobre la acción.

d) La programación de las Unidades: un diseño abierto y cambiante Durante el Máster en Formación del Profesorado de Enseñanza Secundaria y Bachillerato en la Universidad Autónoma de Madrid, y más concretamente en la asignatura de Didáctica de la Educación Física, realizamos muchas prácticas sobre las leyes educativas. Leíamos los objetivos planteados por el BOE, después las concreciones del BOCM y a partir de ahí tratábamos de redactar objetivos específicos para una sesión. La lógica de actuación se basaba en no dejar ni un solo objetivo sin reflejo legal, ni tampoco ninguna actividad sin programar al diseñar la Unidad Didáctica. Desde el primer momento, el profesor debía tener decidido el número de sesiones de la UD y, para cada sesión, los ejercicios, su progresión, su temporalización y sus variantes. 
Ya para empezar me sorprende el término "Proyecto de Unidad Didáctica". En mi colegio a esto se le llama "Unidad Didáctica". Sin más. Lo primero que me viene a la cabeza es: "vaya, ya se ha confundido A. al enviarme la UD y solo me ha enviado el principio, la parte más general". Y le escribo pidiéndole el resto, a lo que me responde que no hay un resto. Que ellos trabajan de esta forma, tratando de "no cerrar las Unidades Didácticas como hacen las editoriales". El fantasma de la frustración y la desesperación aparece en mi mente. ¿Cómo voy a replicar en mi colegio una Unidad Didáctica si no tengo más que unas cuantas páginas llenas de teoría, buenas intenciones, algún anexo de instrumento de evaluación y referencias bibliográficas? (Extracto de mi primer post en (Re)Produce. 22 de noviembre de 2013)

En (Re)Produce se usa la expresión "Proyecto de Unidad Didáctica" para referirse a lo que yo siempre denominé "Unidad Didáctica". Esto ya dice algo sobre las diferencias. Al comienzo me parece imposible poder replicar la UD de "Juego Bueno" sin referencias explícitas a las actividades propuestas para cada sesión y sin saber siquiera el número de sesiones de las que consta la UD. Mi opinión, al principio de este proceso, era la siguiente:

No me encuentro en condiciones de señalar si es "mejor" o "peor" que las Unidades Didácticas estén más o menos cerradas en el papel. No quiero tomar ninguna decisión aún. Quiero ver cómo transcurre este curso al tratar de replicar las UD de P., al recibir feedback de mi comunidad de práctica y al leer y profundizar en la filosofía del Tratamiento Pedagógico de lo Corporal. (Extracto de mi primer post en (Re)Produce. 22 de noviembre de 2013).

Desde este punto se observan numerosas preguntas en las que busco orientación práctica para llevar a cabo la Unidad Didáctica. Algunos de los elementos por los que pregunto, al no estar explicitados en la Unidad Didáctica, son:

- Sobre la evaluación

Me gustaría pasar una hoja de evaluación de la Unidad Didáctica a los alumnos, para poder tener información de cómo la han vivido, qué aprendizajes han obtenido, qué elementos han sido importantes para ellos, etc... Esta evaluación la haré al terminar la Unidad Didáctica. (Comentario en (Re)Produce, noviembre 2013)

- Sobre los objetivos de las UD

He dejado en un post un poco más arriba una duda acerca de cómo conseguir el objetivo "Conocer el procedimiento del método experimental". ¿Alguien puede ayudarme con esto? (Comentario en (Re)Produce, diciembre 2013)

- Sobre la progresión de los contenidos

Después de que hayamos terminado con la parte de los juegos diseñados por los alumnos, ¿qué sesión o sesiones se os ocurren para cerrar la Unidad Didáctica? ¿Una sesión en la que trabajemos sobre la modificación de los diferentes elementos del juego para ver qué ocurre? ¿Una sesión más teórica o teórico-práctica? (Comentario en (Re)Produce, diciembre 2013) 
- Número de sesiones de la UD

Buenos días. Leyendo la UD me ha surgido la misma duda que con las anteriores. ¿Cómo se decide el número de sesiones de la UD? ¿Y cuándo se toma esta decisión?¿Qué preguntas creéis que puedo hacer? (Comentario en (Re)Produce, marzo 2014)

- Sobre las actividades

Leyendo la UD me surge una duda: ¿La UD está pensada para basarla en Polis y Cacos solamente, o también en otros juegos de invasión? (Comentario en (Re)Produce, abril 2014)

- Sobre los emparejamientos

Leyendo el plan de lección 1, me surge una duda: ¿Qué hacemos para formar las parejas para la "representación final de la sesión"? En el resto de ejercicios van cambiando de parejas todo el tiempo, pero en este último trabajan únicamente con una persona. ¿Dejo que sean los alumnos los que se emparejen? ¿Hago yo las parejas? ¿Preferiblemente mixtas? ¿O no? (Comentario en (Re)Produce, febrero 2014)

Estas dudas que me surgen al inicio de cada Unidad Didáctica a partir de la lectura de los proyectos de UD o de los planes de lección elaborados por P. son las que inician en casi todos los hilos los primeros debates. El carácter abierto de las programaciones da lugar a interpretaciones diferentes y a propuestas también variadas.

Lo que sí es del TPC es que se entiende que esa idea general de UD debe ser concretada en cada contexto según las necesidades particulares (Corrección de participante 2, marzo 2016)

Quizá en el comentario anterior está la clave de este elemento del TPC. Las Unidades Didácticas son abiertas en su programación para dejar la labor de concretar a los docentes en cada centro educativo e, incluso, en cada grupo del centro educativo. Se huye, por lo tanto, de crear una Unidad Didáctica tan cerrada que no deje lugar a las particularidades de cada contexto.

\section{Conclusiones}

Mediante el estudio de mi trayectoria formativa puede comprobarse que ésta está desprovista por completo de cualquier indicio de elementos propios del TPC. Por tanto, no será hasta que comienzo a participar activamente en Multiscopic, dentro del grupo (Re)Produce, que los primeros rasgos de esta corriente comienzan a hacerse presentes. La asimilación de los principales elementos del TPC no se produce, en mi caso, de forma inmediata, sino que necesito el paso de los meses y el transcurso de las unidades didácticas para ir leyendo los comentarios de los participantes en la comunidad y entresacando aquellos valores o conceptos reiterados que termino aceptando como parte del cuerpo teórico del TPC. Los elementos más destacables del TPC con los que me encuentro y convivo durante los dos cursos de participación en (Re)Produce, y que también se presentan en la Figura 1, son: 
- La desaparición de la dualidad cuerpo-mente

- La consciencia sobre el aprendizaje

- El docente como provocador de situaciones de reflexión

- La programación de las Unidades: un diseño abierto y cambiante

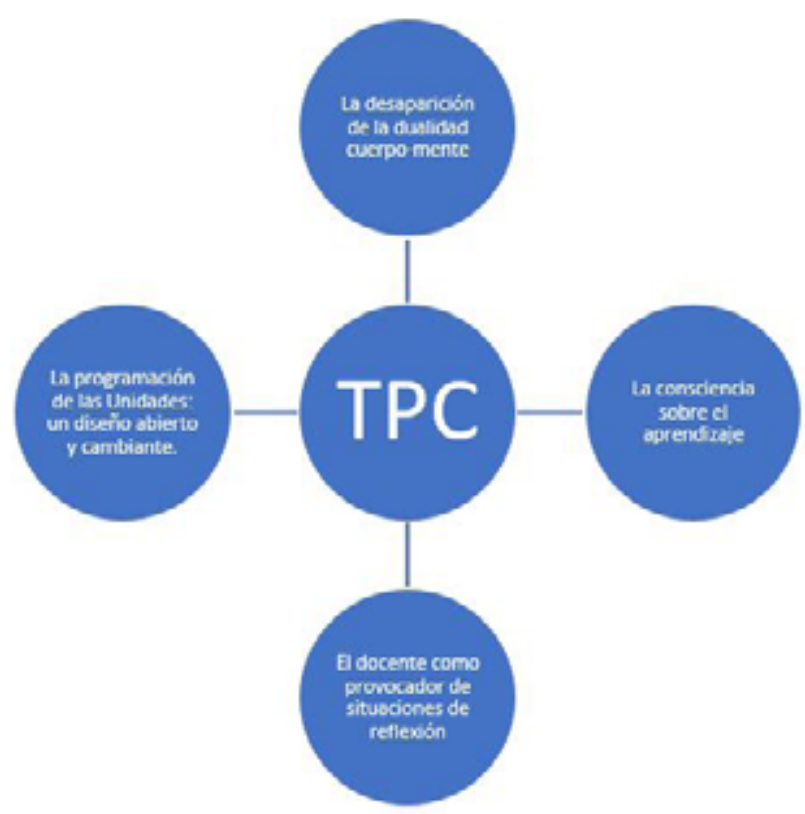

Figura 1. Principales aprendizajes del investigador al acercarse alTPC mediante MultiScopic. Fuente: elaboración propia.

El método para ir comprendiendo el TPC no es sistemático, ni tampoco trato conscientemente de utilizar un mecanismo formal para conocer esta corriente. A través de los diálogos que se producen en la comunidad de práctica virtual voy de la superficie a la profundidad de esta forma de entender la Educación Física.

Como parte del proceso de replicación de las Unidades Didácticas, introduzco los elementos del TPC a mis prácticas diarias en el aula, dentro del contexto de exposición a los comentarios de la comunidad de práctica en (Re)Produce, que me guía y me aconseja en aquellos aspectos complicados para mí, por mi condición de novato, y me confirma en otros elementos que voy interiorizando como parte de mi constructo ideológico que se visualiza en las lecciones de Educación Física. 


\section{Referencias bibliográficas}

Armour, K., y Yelling, M.R. (2004). Professional development and professional learning: Bridging the gap for experienced Physical Education teachers. European Physical Education Review, 10, 71-94. doi: 10.1177/1356336X04040622

Blumer, H. (1982). El interaccionismo simbólico perspectiva y método. Barcelona: Hora S.A.

Bores García, D., González Calvo, G., y García Monge, A. (2018). (Re)Produce: Desarrollo profesional docente en una comunidad de práctica virtual informal de educación física. Sportis Science Journal, 4(3), 480-507. doi: 10.17979/sportis.2018.4.3.3301

Bores Calle, N. (Ed.) (2005). La lección de Educación Física en el Tratamiento Pedagógico de lo Corporal. INDE, Barcelona.

Bores Calle, N. (2006). El cuaderno del alumno en el área de Educación Física en la Enseñanza Secundaria Obligatoria (Tesis Doctoral). España: Universidad de Valladolid.

Bruner, J. (1996). Frames for thinking. Ways of Making Meaning. En D.R. Olson y N. Torrance (Eds.), Modes of thought. Explorations in culture and cognition (pp. 93-105). Cambridge: Cambridge University Press.

Devís Devís, J., y Sparkes, A. (2004). La crisis de identidad de un estudiante universitario de Educación Física: la reconstrucción de un estudio biográfico. En A. Sicilia Camacho y J. M. Fernández Balboa (Eds.), La otra cara de la investigación. Reflexiones desde la Educación Física (pp. 83-106). Sevilla: Wanceulen.

García Monge, A., González Calvo, G., y Bores García, D. (2018). "I like the idea but" ... the gap in participation in a virtual community of practice for analyzing physical education. Open Learning: The Journal of Open, Distance and e-Learning. doi:10.1080/02680513.2 018.1505486

Generelo, E. (1995). Seguimiento del compromiso fisiológico en una clase de deporte educativo en las primeras edades de educación primaria. Tesis inédita. Universidad de Zaragoza, Zaragoza.

González Calvo, G., y Bores García, D. (2014). Estudiando el proceso de configuración de MultiScopic, una red on-line profesional de análisis de la práctica de Educación Física. Educación Física y Deporte, 33(2), 233-258. doi: 10.17533/udea.efyd.v33n2a02

González Calvo, G., y Arias Carballal, M. (2017). A teacher's personal-emotional identity and its reflection upon the development of his professional identity. The Qualitative Report, 22(6), 1693-1709.

González Calvo, G., y Fernández Balboa, J.M. (2016). A qualitative analysis of the factors determining the quality of relations between a novice physical education teacher and his students' families: implications for the development of professional identity. Sport, Education and Society, 23(5), 491-504. doi:10.1080/13573322.2016.1208164

González Calvo, G. (2013). Evolución de la identidad profesional de un docente novel de Educación Física: análisis a partir de la reflexión de sus experiencias personales y de su propia práctica. Tesis Doctoral. Universidad de Valladolid, Valladolid. 
Goodson, I. (2004). Historias de vida del profesorado. Barcelona: Octaedro-EUB.

Lave, J. y Wenger, E. (1991). Situated learning: Legitimate peripheral participation. Cambridge, UK: Cambridge University Press. doi: 10.1017/CBO9780511815355

Martínez Álvarez, L., Bores Calle, N., García Monge, A., Barbero González, J.I., Vaca Escribano, M., Abardía Colás, F., Hernández Martín, A., Miguel Aguado, A., y Rodríguez Campazas, H. (2009). Una perspectiva escolar sobre la Educación Física: buscando procesos y entornos educadores. En L. Martínez Álvarez y R. Gómez (Coords.), La Educación Física y el deporte en edad escolar: el giro reflexivo en la enseñanza (pp. 137-167). Miño y Dávila: Buenos Aires.

Merton. (1980). Teoría y estructura sociales. México: Fondo de Cultura Económica.

Nias, J. (1989). Primary teachers talking: A study of teaching as work. London: Routledge.

Schön, D. (1998). El profesional reflexivo. Cómo piensan los profesores cuando actúan. Barcelona: Paidós.

Segelström, F., y Holmlid, S. (2015). Ethnography by design: On goals and mediating artefacts. Arts and Humanities in Higher Education: An International Journal of Theory, Research and Practice, 14(2), 134-149.

Sierra, A. (2000). Influencia de un programa de prácticas para la formación del maestro especialista en Educación Física sobre el compromiso fisiológico del alumnado de educación primaria en clase de Educación Física. Tesis inédita, Universidad de Granada, Granada.

Sierra, A. (2003). Actividad física y salud. El compromiso fisiológico en clase de Educación Física. Sevilla: Wanceulen.

Taylor, C. (1996). Identidad y reconocimiento. Revista Internacional de Filosofía Política, 7, 10-19. Vaca Escribano, M. (2007). Un proyecto para una escuela con cuerpo y en movimiento. Ágora para la EF y el Deporte, 4-5, 91-110.

Vaca Escribano, M. (2010). Teorías y prácticas de calidad en Educación Física: Una unidad (didáctica) de investigación-acción. Ágora para la EF y el Deporte, 12, 289-307.

Wing Lai, K., Pratt, K., Anderson, M., y Stigter, J. (2006). Literature review and synthesis: online communities of practice. A report submitted to the Ministry of Education. Faculty of Education, University of Otago. Dunedin, New Zealand.

Zabalza, M. A. (2008). Diarios de clase: un instrumento de investigación y desarrollo profesional. Madrid: Narcea. 